\title{
Do the hemispheres differ in their preparation for global/local processing?
}

\author{
Gregor Volberg • Ronald Hübner
}

\begin{abstract}
It has recently been suggested that hemispheric differences for global and local processing already occur in response to visual pre-cues that direct attention to a specific level. However, in the supporting studies cue information was confounded with the form of the cues. In order to dissolve the confound, we compared event-related brain potentials towards cues differing in form with those towards identically formed color cues. As a result, hemispheric differences were found only for the former cue type. The data thus show that the mere cue information does not produce hemispheric asymmetries associated with global/local target stimulus processing.
\end{abstract}

Keywords Event-related potentials $\cdot$ Hemispheric asymmetry $\cdot$ Hierarchical processing $\cdot$ Attention

\section{Introduction}

It is widely accepted that the local and global levels of hierarchically structured visual stimuli are processed with a higher efficiency in the left and right cerebral hemisphere $(\mathrm{LH} / \mathrm{RH})$, respectively. In a typical study, compound letters like those in Fig. 1 are presented and

G. Volberg $(\bowtie)$

University of Regensburg,

Institute for Experimental Psychology,

Universitätsstraße 31, 93053 Regensburg, Germany

e-mail: gregor.volberg@psychologie.uni-regensburg.de

R. Hübner

University of Konstanz, Psychology,

P.O Box D23, 78457 Konstanz, Germany subjects are required to identify the form at one specific (global or local) level that is indicated by a preceding cue stimulus. Using this procedure, hemispheric asymmetries could be demonstrated with a variety of experimental techniques, including lesion studies (e.g. Robertson and Lamb 1991), response-time studies (e.g. Hübner and Malinowksi 2002), event-related potentials studies (ERP, e.g. Volberg and Hübner 2004), and brain imaging studies (e.g. Heinze et al. 1998).

Yamaguchi et al. (2000) went a step further and examined whether the hemispheres already differ in the preparation for the processing of the upcoming global or local level. To this end, they let their subjects detect letters at a pre-cued level of a compound stimulus and recorded the ERP responses in the interval between cue stimulus and target stimulus. Interestingly, the authors found a stronger $\mathrm{LH}$ than $\mathrm{RH}$ activity in response to cue stimuli for the local level, whereas the pattern was reversed for cue stimuli indicating the global level. The effect arose $240 \mathrm{~ms}$ after cue onset and was most prominent at temporal, parietal and occipital sites. Recently, comparable results were also found in a functional magnetic resonance imaging study by Weissman and Woldorff (2005). These authors found higher activations in the intraparietal sulcus of the LH in response to locally directed cue stimuli than in response to globally directed ones. Altogether, these results suggest that hemispheric differences do not only appear during the processing of global or local target stimulus information, but also during the preparation interval in response to cue stimuli.

However, a shortcoming in these studies considerably limits the interpretation of the data. It is that the cue stimuli employed in the global and local conditions 


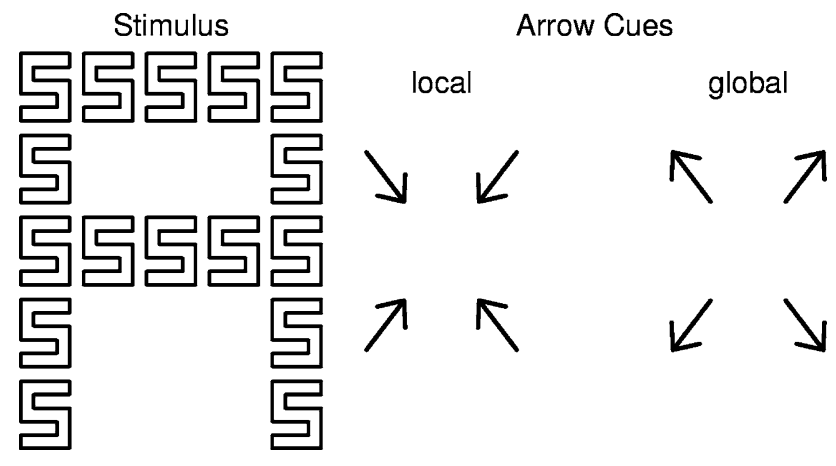

Fig. 1 Arrow cues and a typical example for a target stimulus

had a different shape. In the Yamaguchi et al. (2000) study, the local level was cued by four arrows pointing inwardly to the corners of a small virtual rectangle, whereas four arrows pointing outwardly to the corners of a larger virtual rectangle served for cueing the global level (see Fig. 1). Given these form differences, it cannot be ruled out that the processing of the cue stimulus itself produced the observed asymmetry. A similar confound holds for the study of Weissman and Woldorff (2005), where the letters ' $L$ ' and ' $G$ ' were used for cueing the local and the global levels, respectively. Because the LH is specialized for language processing, the use of letters as cue stimuli is problematic. Indeed, hemispheric differences were restricted to the $\mathrm{LH}$ in that study, possibly as a result of the $\mathrm{LH}$ dominance for language processing.

Thus, it remains unclear whether the observed hemispheric asymmetries in response to cue stimuli were due to specific cue information or due to the associated form differences. Knowing which of these alternatives is true is an important issue for our understanding of hemispheric asymmetries in hierarchical processing, though. This is because the idea of a 'top-down asymmetry', as proposed by Yamaguchi et al. (2000), is partly incompatible with other explanations on hemispheric differences in hierarchical processing. For example, in their integration theory of global and local processing Hübner and Volberg (2005) proposed that the LH and RH mainly differ in their ability to produce high-level representations of compound stimuli where the levels are integrated with their respective contents. Because cues are typically single-level stimuli where no such integration is required, the occurrence of hemispheric differences can not easily be explained. It also contradicts the view that the hemispheres are specialized for processing low and high spatial frequencies carrying the global or the local information, respectively (Sergent 1982). Again, since global/local cue information is typically not associated with a low/high spatial frequency range, one would not expect to see the proposed hemispheric specialization. Thus, taken together, the idea that hemispheric asymmetries already occur in response to the level indicated by the cue and before the actual hierarchical stimulus appears is incompatible with present models on global and local processing.

Therefore, in order to test whether cue information or the associated form differences were crucial in the former studies, two different types of cue stimuli were used in the present ERP study. The first type, arrow cues (see Fig. 1), was identical to that used by Yamaguchi et al. (2000). The second type, color cues, were cue stimuli with a fixed rectangular form that signaled the target level by their color. If the different forms of the arrow cues produce hemispheric asymmetries, then they should occur only for this cue type. In contrast, if attention towards a specific level is sufficient, then hemispheric asymmetries should also occur with the color cues.

\section{Method}

Subjects

Subjects were 16 healthy volunteers from the Universität Konstanz who either received course credits or a small fee for participation. Two subjects were excluded from the further analysis due to excessive artifacts in the EEG data. Thus, 14 subjects ( 11 females, 3 males, mean age 24.5 years) remained in the sample. All of them were right-handed by self-report, had normal or corrected-to-normal vision, and gave informed consent prior to the experiment. The experiment was approved by the appropriate ethics committee and was carried out according to the principles laid down in the Helsinki declaration.

\section{Stimuli and apparatus}

The hierarchical target stimuli were constructed from the letters A, S, H, E, O. All combinations could occur except for stimuli with an $\mathrm{O}$ on both levels. Thus, there were 24 hierarchical target stimuli. Global letters were constructed from identical local letters in a $5 \times 5$ grid, where the local letters were drawn as white outlines on a black background. At a viewing distance of $81 \mathrm{~cm}$ the local and the global letters subtended $0.5 \times 0.7^{\circ}$ and $3.3 \times 4.5^{\circ}$ of visual angle, respectively.

As cue stimuli served either four inwardly or outwardly pointing arrows, or the outline of a red or a blue rectangle. The cue stimuli had half the size of the global target stimulus form. All stimuli were presented on 
a 19-inch color monitor with a resolution of $1024 \times 768$ pixels at a vertical refresh rate of $60 \mathrm{~Hz}$. Responses were given on a two-button mouse.

Procedure

A trial sequence started with a central $300 \mathrm{~ms}$ presentation of a cue stimulus signaling the global or local target level. After a cue-stimulus-interval of $800 \mathrm{~ms}$, a target stimulus appeared for $100 \mathrm{~ms}$ in the screen center. The task was a speeded categorization of the letter that occurred at the level indicated by the cue stimulus. To this end, subjects pressed an associated button with the index or middle finger of the same hand. The letters $\mathrm{A}$ and $\mathrm{S}$ and the letters $\mathrm{H}$ and $\mathrm{E}$ were associated with one common response button, respectively, whereas the form $\mathrm{O}$ required no response. After the button press, the next trial started with a delay from 1,000 to $1,300 \mathrm{~ms}$. Two factors were varied in the experiment: Cue Type (arrow and color) and Target Level (global and local). Whereas the target level was randomized, the cue type was changed as a blocked variable between two sessions that were ran on consecutive days. Each session was preceded by two training blocks. Altogether, the subjects performed 16 experimental blocks of 96 trials. Response hand, starting condition of the cue type, mapping of color and level in the color cue condition, and assignment of letters to response buttons were counterbalanced across subjects.

\section{ERP recording}

The continuous EEG was recorded with a 128 channel EGI sensor net (Electrical Geodesics Inc., Eugene, OR) and referenced to the vertex electrode during recording. Impedance of the electrodes was kept below $50 \mathrm{k} \Omega$. The EEG was amplified with a bandwidth of $0.1-100 \mathrm{~Hz}$ and digitized at a rate of $250 \mathrm{~Hz}$. After recording, a digital low-pass Butterworth filter with $40 \mathrm{~Hz}$ was applied. For artifact correction, the SCADS procedure was used (Junghöfer et al. 2000). The data were arithmetically converted to average reference as recommended in a recent guideline for ERP research (Picton et al. 2000).

Epochs of trials with correct behavioral responses were segmented into intervals of $800 \mathrm{~ms}$, including a $200 \mathrm{~ms}$ pre-onset baseline. The $600 \mathrm{~ms}$ post-stimulus interval covers the time range of the N2 and early P3 components that are typically associated with hemispheric asymmetries in hierarchical processing. Segmentation was performed twice, once locked to the onset of the cue stimulus and once to onset of the tar- get stimulus. Neighboring channels were grouped to seven homologous electrode pairs (FM: frontal medial, FL: frontal lateral, C: central, T: temporal, CP: centro-parietal, P: parietal, and O: occipital; see Fig. 2a).

\section{Statistical analysis of ERP data}

ERPs were quantified by the mean amplitudes within the investigated time ranges, where the amplitudes were measured relative to a pre-cue or pre-stimulus baseline of $200 \mathrm{~ms}$. To achieve a measure of hemispheric asymmetries, the mean amplitudes in local conditions were subtracted from those in global conditions in either hemisphere. Then, the difference wave in the $\mathrm{RH}$ was subtracted from that in the LH. One-sample $t$ tests were applied in order to see whether the mean of the resulting wave differs from zero.

The analysis of cue-locked ERPs was similar to that of Yamaguchi et al. (2000). Per condition and per electrode, the amplitudes were averaged over 30 consecutive $20 \mathrm{~ms}$ intervals from onset of the cue stimulus to the end of the ERP segment. The procedure has the advantage that no a priori hypothesis is needed about the latencies of the resulting ERP components. This reduces the probability that effects are overlooked due to unfavorable averaging. In order to adjust the alpha error for multiple t-test, the procedure described by Guthrie and Buchwald (1991) was used. It reveals the minimum number of consecutive significant comparisons that must be achieved for attaining a family-wise alpha error below $5 \%$. For the present study, a run of three consecutive tests with $\alpha<0.05$ or four consecutive test with $\alpha<0.10$ is required to define an effect with a family wise error $<5 \%$.

Amplitude differences in response to stimuli where measured 80-100 ms (P1), 120-180 ms (N1), 200$300 \mathrm{~ms}(\mathrm{~N} 2)$ and 300-600 ms (P3) after stimulus onset.

\section{Results}

Behavioral data

Reaction times of trials with correct behavioral responses as well as error rates were subjected to an ANOVA for repeated measures with the factors Cue Type (color and arrow) and Target Level (global and local). The analysis revealed no significant results. Especially, it should be noted that response speed and accuracy for stimuli that were preceded by a color cue or by an arrow cue did not differ, $F(1,13)=1.32$, $P>0.27$ and $F(1,13)=0.05, P>0.80$, respectively. This 
Fig. 2 a Grand mean average waveforms in response to cues at centro-parietal $(C P)$, parietal $(P)$, and occipital $(O)$ electrode locations. b Spherical spline-interpolated scalp topographies for the difference wave of local minus global conditions (averaged 300$500 \mathrm{~ms}$ after cue onset, scale given on the left side of the color bar). c Laplacian (SCD) of the topographic maps shown in $\mathbf{b}$, scale given on the right side of the color bar a) Arrow Cues
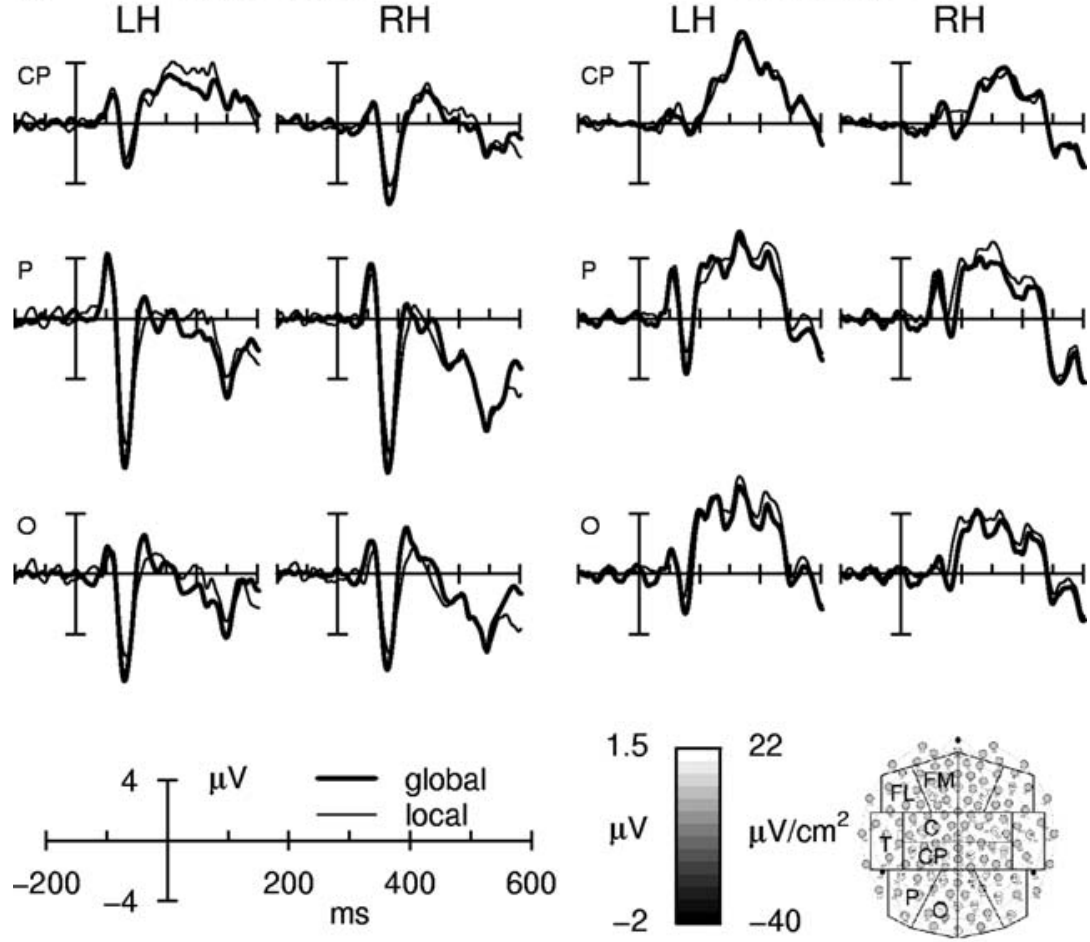

b)

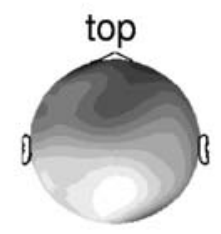

back
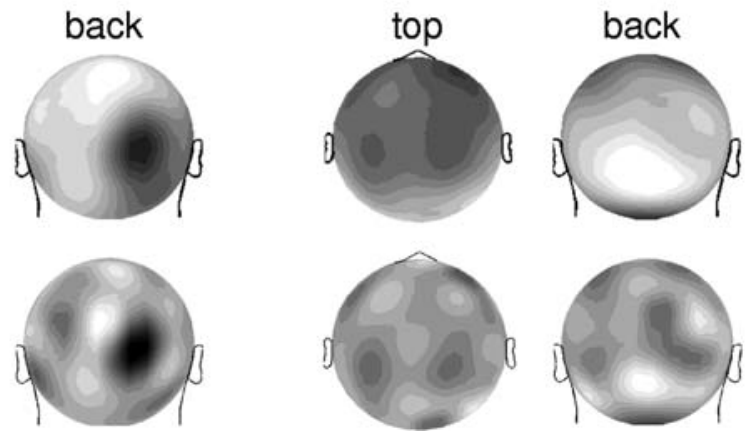

c)
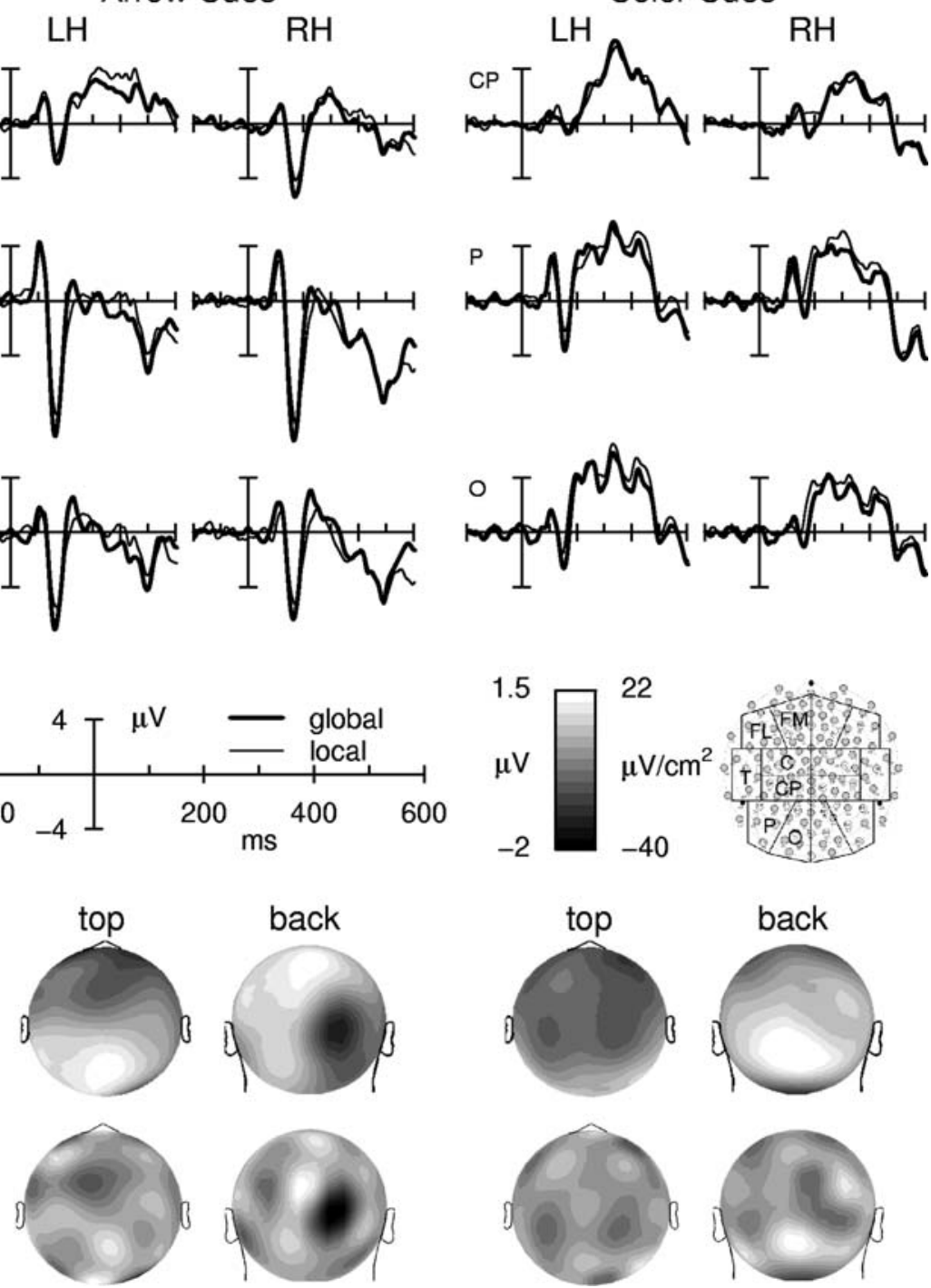

onset of the cue stimulus $[t(13)=2.29-3.67$, all $P<0.05]$. Additionally, the difference was marginally significant 260-280 and 480-500 ms after onset of the cue stimulus, $t(13)=1.82-2.03$, both $P<0.10$. Within the 560-600 ms range, amplitudes were generally larger in the global than in the local condition, but the difference was larger in the RH than in the LH. In all other time ranges, the amplitudes were more positive in response to locally than to globally directed cue stimuli in the $\mathrm{LH}$, and more positive for globally than for locally directed cue stimuli in the $\mathrm{RH}$. Although less pronounced, comparable results were also observed at parietal electrodes. Hemispheric differences were prominent 300-360 ms after onset of the cue stimulus $[t(13)=2.16,1.97$ and 2.89 , $P<0.10,0.10$ and 0.05 , respectively], and again 420 $460 \mathrm{~ms}$ after onset of the cue stimulus $[t(13)=1.86$, $P<0.10$ and $t(13)=2.97, P<0.05]$. As for occipital electrodes, the amplitudes were more positive in the the time range of 320-460 ms after onset of the stimulus, and again 500-540 and 560-600 ms after
As one can see, marked hemispheric differences occurred in the arrow cue condition. They were most pronounced at occipital electrodes, where significant effects showed up in seven consecutive bins covering 
local than in the global condition in the $\mathrm{LH}$, and more positive for the global than for the local condition in the RH.

On contrast, in the color cue condition there was no indication for hemispheric differences. Noticeable results were observed at centroparietal and parietal leads 180-220 ms after cue onset [centroparietal: $t(13)=-1.82$ and -2.01 , both $P<0.10 ;$ parietal: $t(13)=-1.78$ and -2.16 , both $P<0.10]$, and at both frontal electrode pairs $520-560 \mathrm{~ms}$ after cue onset [frontal medial: $t(13)=2.44$ to 2.72 , all $P<0.05$; frontal lateral: $t(13)= \pm-2.31$ to \pm 2.64 , all $P<0.05$ ]. However, none of these differences met the criteria for a significant effect set up with the Guthrie-Buchwald procedure.

The difference between arrow cues and color cues can also be seen in the scalp topography maps of the corresponding difference waves, local minus global condition, averaged $300-500 \mathrm{~ms}$ after cue onset. In the arrow cue condition (Fig. 2b, left panel), there was a positive-going central wave stretching into the posterior regions of the $\mathrm{LH}$ together with a negative deflection in the posterior part of the RH. By contrast, in the color cue condition (Fig. 2b, right panel) no hemispheric difference was observed. In order to reduce the effect of volume conductance, a scalp current density analysis (Laplacian, Fig. 2c) was additionally performed. For arrow cues, it revealed a clear negative focus in the parietal/occipital $\mathrm{RH}$ together with a smaller and more centro-parietally located positive focus in the LH. For color cues, there was one broad positive focus at occipital leads centered at the midline, as well as two weaker negative foci distributed to left and right parietal regions.

Further statistical analyses for the mean amplitudes within the 300-500 ms time range confirmed that picture (data collapsed over parietal and occipital electrodes). Hemispheric differences were present in the arrow cue condition, $t(27)=3.68, P<0.01$, but not in the color cue condition, $t(27)=0.15, P>0.80$. Also, the difference between the cue types with respect to hemispheric asymmetries was significant, $t(27)=2.26$, $\mathrm{P}<0.05$.

\section{ERPs in response to target stimuli}

Significant results were found for the mean P1 amplitudes at centroparietal $[t(13)=-2.99, P<0.05]$, parietal $[t(13)=-3.47, P<0.01]$ and occipital electrodes $[t(13)=-2.41, P<0.05]$. At all sites, the amplitudes were more positive towards local targets than towards global targets in the LH, whereas in the RH they were more positive towards global as compared to local targets. Further effects were not observed. We also evaluated whether target stimulus-related hemispheric differences were modulated by the type of the preceding cue. Generally, this was not the case.

\section{Discussion}

In the present study it was investigated whether the cue information as such or simply form differences between the arrow cues produced hemispheric asymmetries in Yamaguchi et al.'s (2000) study. The data provide a clear answer. In the arrow cue condition, marked hemispheric differences occurred. They emerged about $300 \mathrm{~ms}$ after cue onset and had a broad posterior distribution covering centro-parietal, parietal and occipital electrode locations. Thus, the results were similar to those reported by Yamaguchi et al. (2000). In contrast, in the condition with cue stimuli of identical form but different colors for indicating the target level, there were no hemispheric differences. These data show that global or local cue information alone (and, consequently, the mere preparation for the processing of certain target levels) does not produce hemispheric differences in hierarchical processing.

One objection could be that the form differences of the arrow cues were easier to decode than the color information of the color cues. This could have led to an automatic processing of the arrow cues, which, in turn, was favorable for the occurrence of hemispheric differences. However, more automatic processing should have resulted in faster responses for the arrow cue condition, which was not the case. It is also important to note that the recording sessions were each preceded by two training blocks, so that subjects had sufficient practice with color cues before the actual experiment started. Thus, it is unlikely that automatic versus controlled cue processing played a critical role in our study.

A related problem is that the waveforms for color cues where somewhat contaminated by alpha oscillations that typically occur in rest conditions. This could indicate that subjects were generally more fatigued in the color cue condition. However, ERPs for color and arrow cues were recorded at two different days, and the starting condition was balanced across subjects. Thus, there was no confound between cue type and temporal order of the conditions within the experiment.

Although the ERPs for the cue stimuli were in the main focus of the present study, also the target stimulus-locked data were analyzed. The outcome did not fully comply with the results reported in the literature. Whereas in the majority of studies hemispheric 
asymmetries did not occur earlier than $250 \mathrm{~ms}$ after target stimulus onset (e.g. Heinze et al. 1998), in the present experiment they emerged already in the P1 component. However, we are not the first observing such early asymmetries. Similar effects were also reported by Evans et al. (2000). One factor for P1 asymmetries is possibly the effort for selecting the letter at the target level. As in the Evans et al. (2000) study, in the present experiment the target selection was rather easy because the stimulus always occurred at the same position and the preparation interval was long (800 ms compared to $350 \mathrm{~ms}$ in Malinowski et al. 2002, or to $600 \mathrm{~ms}$ in Volberg and Hübner 2004). Nevertheless, it remains unclear why early lateralization occurs under such conditions.

In any case, and more important for the objective of the present study, the data for the color cue condition indicate that the hemispheres do not differ in their preparation for the processing of a specific stimulus level. This suggests that the effects in the cue-stimulus interval reported by Yamaguchi et al. (2000) were simply due to the confound of the form of the cues with the level they signaled. But why did arrow cues produce hemispheric asymmetries? An obvious difference between the types of cue stimuli is that in the color cue condition, a simple stimulus feature was sufficient for retrieving the cue information. On contrast, in the arrow cue condition the global and local cues both consisted of four identical arrows, so that the arrows as such were not indicative of the target level. It was their spatial arrangement that was crucial. Therefore, we propose that arrow cues had to be processed in a global/local manner in order to extract the relevant information. Consequently, for these cue stimuli the same hemispheric differences show up that usually appear in response to compound target stimuli.

This proposition is related to the integration theory of global/local processing (Hübner and Volberg 2005). The theory says that the hemispheres do not generally differ in their efficiency for processing the information at the global or local stimulus level, but rather in their capacity for binding the stimulus information to their respective level. The present data suggest that this assumption might also hold for more spatial binding processes (Treisman and Gelade 1980). If we consider the arrow cues, then it is obvious that the arrows alone were not sufficient for uniquely signaling the required target level. Rather, it was necessary to bind at least the four arrowheads to their correct spatial position. This resembles the integration of form and stimulus level as proposed by the integration theory of global/ local processing. Also the brain asymmetries evoked by arrow cues are comparable to those we found in earlier global/local studies specifically if an integration of target level and form was necessary (Volberg and Hübner 2004, Malinowksi et al. 2002). In both cases, asymmetries had a centroparietal or parietal topography and were most marked within the early P3 component. This suggests that $\mathrm{P} 3$ asymmetries for arrow cues also reflect activity related to the construction of integrated hierarchical stimulus representations. Further research will have to show how far this analogy holds.

Finally, it should be noted that, although our ERPs did not reveal hemispheric differences in response to cue stimuli, they may be observed if other analyses are used. For example, Doesburg and Ward (2006) found that long-range oscillatory synchrony in EEG gamma and MEG high alpha bands is lateralized in preparation for responding to a target on one or the other side of space. It is conceivable that a similar result can be observed in a synchrony analysis of the preparation for global or local processing.

Acknowledgments This research was supported by a grant from the Deutsche Forschungsgemeinschaft (DFG) to the second author (Hu 432/7). We thank Franka Glöckner for data aquisition.

\section{References}

Doesburg S, Ward LM (2006) Long-distance alpha-band MEG synchronization maintains selective attention. In: Cheyne D, Ross B, Stronik G, Weinberg H (eds) Proceedings of the 15th International Conference on Biomagnetism BIOMAG (in press)

Evans MA, Shedden JM, Hevenor SJ, Hahn MC (2000) The effect of variability of unattended information on global and local processing: evidence for lateralization at early stages of processing. Neuropsychologia 38:225-239

Guthrie D, Buchwald JS (1991) Significance testing of difference potentials. Psychophysiology 28:240-244

Heinze HJ, Hinrichs M, Scholz M, Burchert W, Mangun GR (1998) Neural mechanisms of global and local processing: a combined PET and ERP study. J Cogn Neurosci 10:485-498

Hübner R, Malinowski P (2002) The effect of response competition on functional hemispheric asymmetries for global/local processing. Percept Psychophys 64:1290-1300

Hübner R, Volberg G (2005) The integration of object levels and their content: a theory of global/local processing and related hemispheric differences. J Exp Psychol Hum Percept Perform 31:520-541

Junghöfer M, Elbert T, Tucker DM, Rockstroh B (2000) Statistical control of artifacts in dense array EEG/MEG studies. Psychophysiology 37:523-532

Malinowski P, Hübner R, Keil A, Gruber T (2002) The influence of response competition on cerebral asymmetries for processing hierarchical stimuli revealed by ERP recordings. Exp Brain Res 144:136-139

Picton TW, Bentin S, Berg P, Donchin E, Hillyard SA, Johnson R, Miller GA, Ritter W, Ruchkin DS, Rugg MD, Taylor MJ (2000) Guidelines for using human event-related potentials to study cognition: recording standards and publication criteria. Psychophysiology 37:127-152 
Robertson LC, Lamb MR (1991) Neuropsychological contributions to theories of part/whole organization. Cognit Psychol 23:299-330

Sergent J (1982) The cerebral balance of power: confrontation or cooperation? J Exp Psychol Hum Percep Perf 8:253-272

Treisman AM, Gelade G (1980) A feature-integration theory of attention. Cognit Psychol 12:97-136

Volberg G, Hübner R (2004) On the role of response conflicts and stimulus position for hemispheric differences in global/ local processing: an ERP study. Neuropsychologia 42:18051813

Weissman DH, Woldorff MG (2005) Hemispheric asymmetries for different components of global/local attention occur in distinct temporo-parietal loci. Cereb Cortex 15:870-876

Yamaguchi S, Yamagata S, Kobayashi S (2000) Cerebral asymmetry of the top-down allocation of attention to global and local features. J Neurosci 20(RC72):1-5 